\title{
On Disagreement about Nonperturbative Corrections in Triple-well Potential
}

\author{
Masatoshi Satc* \\ The Institute for Solid State Physics, The University of Tokyo, \\ Kashiwanoha 5-1-5, Kashiwa-shi, Chiba 277-8581, Japan \\ Toshiaki Tanaked \\ Departamento de Física Teórica II, Facultad de Ciencias Físicas, \\ Universidad Complutense, 28040 Madrid, Spain
}

\begin{abstract}
We examine in detail nonperturbative corrections for low lying energies of a symmetric triplewell potential with non-equivalent vacua, for which there have been disagreement about asymptotic formulas and controversy over the validity of the dilute gas approximation. We carry out investigations from various points of view, including not only a numerical comparison of the nonperturbative corrections with the exact values but also the prediction on the large order behavior of the perturbation series, consistency with the perturbative corrections, and comparison with the WKB approximation. We show that all the results support our formulas previously obtained from the valley method calculation beyond the dilute gas approximation.
\end{abstract}

PACS numbers: 03.65.Sq; 02.30.Mv; 11.25.Db

Keywords: triple-well potential; (non)perturbative corrections; instantonic calculation; dilute gas approximation; large order behavior; WKB approximation

*Electronic address: msato@issp.u-tokyo.ac.jp

${ }^{\dagger}$ Electronic address: ttanaka@ciruelo.fis.ucm.es 


\section{INTRODUCTION}

It has been widely known that the spectral splitting of the lowest two states of a quantum mechanical symmetric double-well potential due to the quantum tunneling is successfully calculated by summing up multiple instanton contributions with the dilute gas approximation [1]. On the other hand, it might not have been duly recognized that a naive application of the method to a bit more complicated system in general confronts some new difficulties and hardly yields proper results. In this respect, the problem of a symmetric triple-well potential described essentially by the following function:

$$
V(q)=\frac{1}{2} q^{2}\left(q^{2}-1\right)^{2}
$$

have been recently attracting attention of several research groups. One of the novel features of the above potential over the symmetric double-well potential, besides the obvious difference in the number of the potential wells, comes from the fact that the harmonic frequency of the central well is different from that of the side wells. As a result, there are no degeneracies between the harmonic oscillator spectrum of the central well and the side wells while that of the left and right well completely degenerate with each other. Thus, it is difficult to expect intuitively how the quantum tunneling effect contributes to the each harmonic oscillator spectrum.

To the best of our knowledge, the multi-instanton calculation technique with the aid of the dilute gas approximation was applied to a triple-well potential problem first by Lee et al. 2] and later independently by Casahorrán [3]. Both of their resulting formulas of the lowest three eigenvalues are however peculiar and doubtful in the fact that they do not coincide with the harmonic oscillator spectrum of the each potential well when the instanton contribution due to the quantum tunneling effect is turned off, although both of the authors did not discuss the validity of them nor compare the obtained results with the exact eigenvalues.

A few years ago, we investigated in Ref. [4] a similar problem in a different context, namely, dynamical breaking of $\mathcal{N}$-fold supersymmetry, by means of the valley method [5], which is a generalization of the semi-classical approximation and enables us to calculate nonperturbative correction beyond the dilute gas approximation. Our formulas of the spectrum are completely different from the ones previously obtained in Refs. 2, 3] and we have justified our results by checking consistency with some characteristic features of $\mathcal{N}$-fold supersymmetry discussed earlier in Ref. [6]. We have found that the contribution from the interaction between the instantons plays a crucial role in the calculation in order to yield the correct formulas. Hence, we have asserted that the dilute gas approximation would fail in the case.

Recently, however, Alhendi and Lashin reexamined the triple-well potential problem and carried out a careful calculation of the multiple instanton contribution with the dilute gas approximation [7]. Their results are different from ours but look sensible in the sense that they reduce to the harmonic oscillator spectrum when the instanton corrections tend to zero, in contrast to the ones in Refs. [2, 3]. They also performed a numerical calculation of the corresponding Schrödinger equation and compared numerically their formulas with the exact values. From the comparison, they have claimed the correctness of their formulas and thus the validity of the dilute gas approximation. However, they did not make a comparison with our results nor examine the accuracy of them.

In this letter, considering the present situation described above, we would like to compare the formulas by Alhendi and Lashin in Ref. 7] and ours in Ref. [4] from various points of 
view. In the next section, we first make a numerical comparison of the nonperturbative corrections. In Section [II] we examine the large order behavior of the perturbation series. In Section IV we take into account the perturbative corrections to check the accuracy of the formulas for the ground state. In Section $\nabla$, we carry out the ordinary WKB calculation for the Schrödinger equation to provide another reference for the comparison. Finally, we summarize the results in the last section.

\section{PURELY NONPERTURBATIVE CORRECTIONS}

In Ref. 7], Alhendi and Lashin investigated the following triple-well potential:

$$
H^{\mathrm{AL}}(x ; \omega)=-\frac{1}{2} \frac{\mathrm{d}^{2}}{\mathrm{~d} x^{2}}+\frac{\omega^{2}}{2} x^{2}\left(x^{2}-1\right)^{2} .
$$

They calculated the sum of multiple instanton contributions with the dilute gas approximation and obtained for the lowest three eigenvalues,

$$
\begin{aligned}
& E_{0}^{\mathrm{AL}}(\omega)=\omega\left(\frac{3}{4}-\frac{1}{4} \sqrt{1+\frac{1024}{3 \pi} \omega \mathrm{e}^{-\omega / 2}}\right), \\
& E_{1}^{\mathrm{AL}}(\omega)=\omega \\
& E_{2}^{\mathrm{AL}}(\omega)=\omega\left(\frac{3}{4}+\frac{1}{4} \sqrt{1+\frac{1024}{3 \pi} \omega \mathrm{e}^{-\omega / 2}}\right) .
\end{aligned}
$$

On the other hand, the triple-well potential we investigated in Ref. [4] is the following:

$$
H^{\mathrm{ST}}(q ; g, \epsilon)=-\frac{1}{2} \frac{\mathrm{d}^{2}}{\mathrm{~d} q^{2}}+\frac{1}{2} q^{2}\left(1-g^{2} q^{2}\right)^{2}+\frac{\epsilon}{2}\left(1-3 g^{2} q^{2}\right)
$$

Utilizing the valley method, we obtained for all the $n_{0}$ th eigenstates localized around the central well and the $n_{ \pm}$th eigenstates with parity \pm localized around the side wells ${ }^{1}$,

$$
\begin{aligned}
& E_{n_{0}}^{\mathrm{ST}}(g, \epsilon)=n_{0}+\frac{1}{2}+\frac{\epsilon}{2}+\frac{\sqrt{2}}{\pi g^{2}} \mathrm{e}^{-1 / 2 g^{2}} E_{n_{0}}^{(2)}(g, \epsilon)+O\left(\mathrm{e}^{-1 / g^{2}}\right), \\
& E_{n_{ \pm}}^{\mathrm{ST}}(g, \epsilon)=2 n_{ \pm}+1-\epsilon+\frac{\sqrt{2}}{\pi g^{2}} \mathrm{e}^{-1 / 2 g^{2}} E_{n_{ \pm}}^{(2)}(g, \epsilon)+O\left(\mathrm{e}^{-1 / g^{2}}\right),
\end{aligned}
$$

where the coefficients $E_{n_{0}}^{(2)}$ and $E_{n_{ \pm}}^{(2)}$ are given by

$$
\begin{aligned}
& E_{n_{0}}^{(2)}(g, \epsilon)=-\frac{1}{n_{0} !}\left(\frac{2}{g^{2}}\right)^{n_{0}}\left(-\frac{1}{g^{2}}\right)^{n_{0} / 2-1 / 4+3 \epsilon / 4} \Gamma\left(-\frac{n_{0}}{2}+\frac{1}{4}-\frac{3}{4} \epsilon\right) \\
& E_{n_{ \pm}}^{(2)}(g, \epsilon)=-\frac{(-1)^{(1-3 \epsilon) / 2} \pm 1}{n_{ \pm} !}\left(\frac{2}{g^{2}}\right)^{2 n_{ \pm}+1 / 2-3 \epsilon / 2}\left(\frac{1}{g^{2}}\right)^{n_{ \pm}} \Gamma\left(-2 n_{ \pm}-\frac{1}{2}+\frac{3}{2} \epsilon\right) .
\end{aligned}
$$

\footnotetext{
${ }^{1}$ Except for the cases $\epsilon= \pm(2 \mathcal{N}+1) / 3(\mathcal{N}=0,1,2, \ldots)$ where a part of the harmonic oscillator spectra of the central and side wells degenerates.
} 
In order to compare the two results, we must first establish the relation between the Hamiltonians Eqs. (2) and (41). By applying a scale transformation on the coordinate $q$ in Eq. (4), we easily find the following relation:

$$
H^{\mathrm{AL}}(x ; \omega)=\omega H^{\mathrm{ST}}\left(\omega^{1 / 2} x ; \omega^{-1 / 2}, 0\right) .
$$

Therefore, the quantities which we shall make comparison with Eqs. (3) are given by ${ }^{2}$

$$
\begin{aligned}
& E_{0}^{\mathrm{ST}}(\omega)=\omega \operatorname{Re} E_{n_{0}=0}^{\mathrm{ST}}\left(\omega^{-1 / 2}, 0\right)=\omega\left(\frac{1}{2}-\frac{\Gamma(1 / 4)}{\pi} \omega^{3 / 4} \mathrm{e}^{-\omega / 2}+O\left(\mathrm{e}^{-\omega}\right)\right), \\
& E_{1}^{\mathrm{ST}}(\omega)=\omega \operatorname{Re} E_{n_{-}=0}^{\mathrm{ST}}\left(\omega^{-1 / 2}, 0\right)=\omega\left(1-\frac{4}{\sqrt{\pi}} \omega^{3 / 2} \mathrm{e}^{-\omega / 2}+O\left(\mathrm{e}^{-\omega}\right)\right), \\
& E_{2}^{\mathrm{ST}}(\omega)=\omega \operatorname{Re} E_{n_{+}=0}^{\mathrm{ST}}\left(\omega^{-1 / 2}, 0\right)=\omega\left(1+\frac{4}{\sqrt{\pi}} \omega^{3 / 2} \mathrm{e}^{-\omega / 2}+O\left(\mathrm{e}^{-\omega}\right)\right) .
\end{aligned}
$$

Here we note that the nonperturbative corrections in Eqs. (6) are in general complex and the real parts of them should be taken into account as the spectral shifts; the imaginary parts of them are to be canceled with the imaginary parts of the perturbative corrections arising from the Borel singularity in the framework of the valley method, see for more details Refs. [4, 5]. We will later consider the imaginary parts in order to investigate the large order behavior of the perturbation series in the next section.

For the purpose of examining the accuracy of the purely nonperturbative corrections in Eqs. (3) and (8), it is important to note that in addition to the nonperturbative corrections estimated in the formulas there are perturbative corrections to the harmonic oscillator spectra $^{3}$. Hence it does not make sense to compare directly them to the exact eigenvalues without taking into account the perturbative contributions. Fortunately, however, the perturbative corrections to the harmonic oscillator spectrum for the first and second excited states are completely the same (cf. Section III). As a result, the difference of the eigenvalues between the first and second excited states $\Delta E_{21}=E_{2}-E_{1}$ only contains the purely nonperturbative contributions. Therefore, the comparison of the quantity $\Delta E_{21}$ enables us to study the accuracy of Eqs. (3) and (8) adequately. In Table In we show i) the exact results $\Delta E_{21}^{e x}$ obtained by solving numerically the Schrödinger equation for the Hamiltonian (2) presented in Ref. [7], ii) $\Delta E_{21}^{\mathrm{AL}}=E_{2}^{\mathrm{AL}}-E_{1}^{\mathrm{AL}}$ obtained from Eq. (31) and the ratios $\Delta E_{21}^{\mathrm{AL}} / \Delta E_{21}^{e x}$, and iii) $\Delta E_{21}^{\mathrm{ST}}=E_{2}^{\mathrm{ST}}-E_{1}^{\mathrm{ST}}$ obtained from Eq. (8) and the ratios $\Delta E_{21}^{\mathrm{ST}} / \Delta E_{21}^{e x}$.

From Table I, we see that our results $\Delta E_{21}^{\mathrm{ST}}(\omega)$ are in better agreement with the numerical exact values $\Delta E_{21}^{e x}(\omega)$ than those by Alhendi and Lashin $\Delta E_{21}^{\mathrm{AL}}(\omega)$. We also find that as the parameter $\omega$ becomes larger (the coupling g becomes smaller), the accuracy of our results becomes better while that of the results by Alhendi and Lashin becomes worse. This suggests that our method provides a reliable semi-classical approximation but the dilute gas

\footnotetext{
${ }^{2}$ In this case, the spectral splitting takes place between the first and second excited states and hence the parity odd state is lower than the parity even state in the spectrum, which is in contrast to the case of symmetric double-well potentials.

${ }^{3}$ In this article, a contribution is called perturbative (nonperturbative) if it can (cannot) be expressed as a (formal) power series in $g=\omega^{-1 / 2}$, respectively. See also Section IV
} 
TABLE I: Comparison of the energy difference between the first and second excited states.

\begin{tabular}{rl|lc|lc}
\hline$\omega$ & $\Delta E_{21}^{e x}(\omega)$ & $\Delta E_{21}^{\mathrm{AL}}(\omega)$ & $\Delta E_{21}^{\mathrm{AL}} / \Delta E_{21}^{e x}$ & $\Delta E_{21}^{\mathrm{ST}}(\omega)$ & $\Delta E_{21}^{\mathrm{ST}} / \Delta E_{21}^{e x}$ \\
\hline 30 & $4.7230 \cdot 10^{-3}$ & $3.7381 \cdot 10^{-3}$ & 0.79147 & $6.8061 \cdot 10^{-3}$ & 1.4411 \\
50 & $9.1006 \cdot 10^{-7}$ & $4.7154 \cdot 10^{-7}$ & 0.51814 & $1.1081 \cdot 10^{-6}$ & 1.2176 \\
70 & $1.0186 \cdot 10^{-10}$ & $4.1959 \cdot 10^{-11}$ & 0.41193 & $1.1667 \cdot 10^{-10}$ & 1.1454 \\
90 & $8.9504 \cdot 10^{-15}$ & $3.1490 \cdot 10^{-15}$ & 0.35183 & $9.9282 \cdot 10^{-15}$ & 1.1092 \\
110 & $6.8449 \cdot 10^{-19}$ & $2.1356 \cdot 10^{-19}$ & 0.31201 & $7.4439 \cdot 10^{-19}$ & 1.0875 \\
\hline
\end{tabular}

approximation does not. As is easily seen from the Euclidean action of the present model

$$
S^{\mathrm{ST}}=\frac{1}{g^{2}} \int \mathrm{d} \tau\left(\frac{1}{2} \dot{x}^{2}+\frac{1}{2} x^{2}\left(1-x^{2}\right)^{2}\right), \quad x=g q,
$$

the coupling constant $g^{2}$ plays a similar role to the Plank constant $\hbar$. Therefore, any reliable semi-classical approximation should work better as the coupling constant $g$ becomes smaller.

\section{LARGE ORDER BEHAVIOR OF PERTURBATION SERIES}

In spite of the fact that perturbation series are in general divergent and at most asymptotic, they contain much information on the property of the physical quantity under consideration. An intimate relation between nonperturbative property and large order behavior of perturbation series is a typical example [8]. In this section, we make an examination from this point of view.

In order to evaluate the perturbative corrections to the harmonic oscillator spectrum of the each potential well, it is convenient to begin with the Hamiltonian (4) and then make the transformation indicated by the scale relation (77) after the perturbative calculation. The coefficients of the perturbation series are systematically calculated with the aid of the Bender-Wu method [9]. First, the perturbation theory around the harmonic oscillator states of the central potential well is set up by decomposing the Hamiltonian into the harmonic oscillator part and the remaining part:

$$
H^{\mathrm{ST}}(q ; g, 0)=-\frac{1}{2} \frac{\mathrm{d}^{2}}{\mathrm{~d} q^{2}}+\frac{1}{2} q^{2}-g^{2} q^{4}+\frac{1}{2} g^{4} q^{6} .
$$

The perturbative corrections to the eigenvalues and eigenfunctions are defined by the following formal series expansions:

$$
E(g)=\sum_{m=0}^{\infty} g^{2 m} c^{[2 m]}, \quad \psi(q ; g)=\mathrm{e}^{-q^{2} / 2} \sum_{k=0}^{\infty} g^{2 k} \sum_{l=0}^{\infty} a_{2 l+P}^{[2 k]} q^{2 l+P}
$$

where $P=0$ (1) for the even (odd) parity states, respectively. For the lowest state, $c^{[0]}=1 / 2$ and $a_{2 l}^{[0]}=0$ for all $l>0$. Requiring that they satisfy the Schrödinger equation, we obtain a 
recursion relation for $a_{2 l+P}^{[2 k]}$ and $c^{[2 m]}$ :

$$
\begin{aligned}
(4 l+1+2 P) a_{2 l+P}^{[2 k]}-(2 l+2 & +P)(2 l+1+P) a_{2(l+1)+P}^{[2 k]} \\
& -2 a_{2(l-2)+P}^{[2(k-1)]}+a_{2(l-3)+P}^{[2(k-2)]}=2 \sum_{m=0}^{k} c^{[2 m]} a_{2 l+P}^{[2(k-m)]}
\end{aligned}
$$

Second, the perturbation theory around the harmonic oscillator states of the side potential wells is defined by shifting the origin of the coordinate to one of the minimum of the side potentials $q \rightarrow q \pm 1 / g$ and then decomposing the Hamiltonian:

$$
H^{\mathrm{ST}}(q \pm 1 / g ; g, 0)=-\frac{1}{2} \frac{\mathrm{d}^{2}}{\mathrm{~d} q^{2}}+\frac{4}{2} q^{2} \pm 6 g q^{3}+\frac{13}{2} g^{2} q^{4} \pm 3 g^{3} q^{5}+\frac{1}{2} g^{4} q^{6}
$$

The perturbative corrections are similarly introduced by ${ }^{4}$

$$
E(g)=\sum_{m=0}^{\infty} g^{2 m} c^{[2 m]}, \quad \psi(q ; g)=\mathrm{e}^{-q^{2}} \sum_{k=0}^{\infty} g^{k} \sum_{l=0}^{\infty} a_{l}^{[k]} q^{l}
$$

The recursion relation for $c^{[m]}$ and $a_{l}^{[k]}$ in this case is then given by

$$
\begin{aligned}
& (4 l+2) a_{l}^{[k]}-(l+2)(l+1) a_{l+2}^{[k]} \pm 12 a_{l-3}^{[k-1]} \\
& +13 a_{l-4}^{[k-2]} \pm 6 a_{l-5}^{[k-3]}+a_{l-6}^{[k-4]}=2 \sum_{m=0}^{[k / 2]} c^{[2 m]} a_{l}^{[k-2 m]}
\end{aligned}
$$

For the lowest state, $c^{[0]}=1$ and $a_{l}^{[0]}=0$ for all $l>0$.

On the other hand, as we have mentioned previously, the imaginary parts of the nonperturbative contributions are to be canceled with those of the perturbative ones in the framework of the valley method. This leads to the following dispersion relation [4, 5] :

$$
c^{[2 m]}=-\frac{1}{\pi} \int_{0}^{\infty} \mathrm{d} g^{2} \frac{\operatorname{Im} E_{\mathrm{NP}}(g)}{g^{2 m+2}} .
$$

This relation enables us to predict the large order behavior of the perturbation series for the eigenvalues. For the lowest three states $\left(n_{0}=n_{ \pm}=0\right)$ in the present case $(\epsilon=0)$, we obtain from Eqs. (15)-(6) and (16)

$$
\begin{aligned}
& c_{0}^{[2 m]} \sim-\frac{2^{5 / 4}}{\pi \Gamma(3 / 4)} 2^{m} \Gamma\left(m+\frac{3}{4}\right) \equiv \bar{c}_{0}^{[2 m]}, \\
& c_{1(2)}^{[2 m]} \sim-\frac{8 \sqrt{2}}{\pi^{3 / 2}} 2^{m} \Gamma\left(m+\frac{3}{2}\right) \equiv \bar{c}_{1(2)}^{[2 m]}
\end{aligned}
$$

\footnotetext{
${ }^{4}$ Here we note that all the perturbative coefficients of odd powers in $g$ for the spectrum vanish due to the parity symmetry of the original Hamiltonian (10).
} 
TABLE II: The ratios of the exact values of the perturbative coefficients $c^{[2 m]}$ to the predicted asymptotic values $\bar{c}^{[2 m]}$.

\begin{tabular}{rll}
\hline$m$ & $c_{0}^{[2 m]} / \bar{c}_{0}^{[2 m]}$ & $c_{1(2)}^{[2 m]} / \bar{c}_{1(2)}^{[2 m]}$ \\
\hline 20 & 0.8946472445 & 0.7797002850 \\
40 & 0.9493285320 & 0.8904365552 \\
60 & 0.9665686152 & 0.9268736279 \\
80 & 0.9750492671 & 0.9451085558 \\
100 & 0.9800964967 & 0.9560611732 \\
120 & 0.9834448543 & 0.9633690036 \\
140 & 0.9858286759 & 0.9685922139 \\
160 & 0.9876123158 & 0.9725115700 \\
180 & 0.9889971178 & 0.9755611660 \\
200 & 0.9901034160 & 0.9780016290 \\
220 & 0.9910075563 & 0.9799989041 \\
240 & 0.9917603143 & 0.9816636733 \\
260 & 0.9923967744 & 0.9830725934 \\
280 & 0.9929419564 & 0.9842804381 \\
300 & 0.9934141831 & 0.9853273870 \\
\hline
\end{tabular}

Therefore, we can check the validity of the results (5) -(6) by comparing the predicted asymptotic forms $\bar{c}^{[2 m]}$ in Eqs. (17) with the exact perturbative coefficients $c^{[2 m]}$ calculated using the recursion relations (12) and (15).

In Table II] we show the ratios $c^{[2 m]} / \bar{c}^{[2 m]}$ up to the order $m=300$. We easily see that the exact values indeed tend to the predicted asymptotic values for both the ground and excited states and thus confirm the correctness of our formulas, at least, for their imaginary parts.

\section{INTERPLAY BETWEEN NONPERTURBATIVE AND PERTURBATIVE CORRECTIONS}

In Section III, we have investigated the nonperturbative corrections for the excited states and confirmed that our formulas obtained from the valley method calculation are more accurate than the ones obtained from the instanton calculation with the dilute gas approximation. Although the analysis of the large order behavior in the previous section ensures the correctness of the imaginary parts of our formulas for both the ground and excited states, it does not necessarily mean that the real part of our formula for the ground state is also correct. In order to check the accuracy of the nonperturbative spectral shift for the ground state we must resort to other means.

As we have mentioned previously, there exist perturbative corrections to the harmonic oscillator spectrum in addition to nonperturbative ones. Hence, each of the spectrum is 
formally expressed as

$$
E(g)=E^{(0)}+E_{\mathrm{NP}}(g)+E_{\mathrm{P}}(g),
$$

where $E^{(0)}$ denotes the harmonic oscillator spectrum when $g=0, E_{\mathrm{NP}}$ the purely nonperturbative part which cannot be represented by a formal power series in $g^{2}=\omega^{-1}$, and $E_{\mathrm{P}}$ the remaining perturbative part. The decomposition (18) suggests that we can check the formulas for the nonperturbative corrections by examining the prediction on the perturbative corrections instead. That is, we can regard each of the following quantity as the prediction of the each formula on the perturbative contribution to the ground state:

$$
E_{\mathrm{P}}^{\mathrm{AL}}(\omega) \equiv E_{0}^{e x}(\omega)-E_{0}^{\mathrm{AL}}(\omega), \quad E_{\mathrm{P}}^{\mathrm{ST}}(\omega) \equiv E_{0}^{e x}(\omega)-E_{0}^{\mathrm{ST}}(\omega),
$$

where $E_{0}^{e x}(\omega)$ is the exact eigenvalue for the ground state, $E_{0}^{\mathrm{AL}}(\omega)$ and $E_{0}^{\mathrm{ST}}(\omega)$ are respectively given by Eqs. (3a) and (8a), both of which consist of the harmonic oscillator eigenvalue and the predicted nonperturbative contribution.

TABLE III: Predicted perturbative contributions to the ground state energy.

\begin{tabular}{rll}
\hline$\omega$ & $E_{\mathrm{P}}^{\mathrm{AL}}(\omega)$ & $E_{\mathrm{P}}^{\mathrm{ST}}(\omega)$ \\
\hline 30 & $-0.818251 \cdots$ & $-0.821854 \cdots$ \\
50 & $-0.7883965537 \cdots$ & $-0.7883970101 \cdots$ \\
70 & $-0.776334145610396 \cdots$ & $-0.776334145651123 \cdots$ \\
90 & $-0.7700548367611102506 \cdots$ & $-0.7700548367611133127 \cdots$ \\
110 & $-0.7661975631223371558989 \cdots$ & $-0.7661975631223371561068 \cdots$ \\
\hline
\end{tabular}

In Table III. we show the numerical values of the predicted perturbative contributions to the ground state energy for each value of $\omega$ calculated using Eq. (19). For the exact eigenvalues $E_{0}^{e x}(\omega)$, we have used again the numerical results shown in Ref. 7].

To examine the accuracy of these predictions, we shall evaluate the exact perturbative contribution $E_{\mathrm{P}}$ by using the perturbation series. Although the perturbation series is generally divergent, as we have already observed in the previous section (cf., Eqs. (17)), the asymptotic property of the perturbation series nevertheless ensures that for a sufficiently small value of the expansion parameter a partial sum of the first finite terms in the perturbation series gives an asymptotic value of the perturbative correction ${ }^{5}$. As a consequence, however small the value of the expansion parameter is, there exists a critical order $m_{c}$ at which the absolute value of the perturbative correction $\left|g^{2 m} c_{0}^{[2 m]}\right|$ becomes minimum. It is apparent that the asymptotic property is lost and replaced by the divergent one when the order of the perturbation exceeds the critical order $m_{c}$. Therefore, the asymptotic values of

\footnotetext{
${ }^{5}$ We note that the asymptotic property of the perturbation series in general has nothing to do with the Borel summability. We also note that in the quantum mechanical systems there exist no renormalon singularities which originate from the IR and UV divergences in the higher-dimensional quantum field theories.
} 
the exact perturbative corrections we should read from the perturbation series are given by the first finite partial sums up to at most the critical order $m_{c}$.

In Table IV] we illustrate the numerical values obtained from the first finite partial sums of the perturbation series for the ground state. Here we note that from the scaling relation (17) the perturbative quantity $E_{\mathrm{P}}(\omega)$ we should take for the Hamiltonian $H^{\mathrm{AL}}(x ; \omega)$ reads

$$
E_{\mathrm{P}}(g)=\sum_{m=1}^{M} g^{2 m} c_{0}^{[2 m]} \longmapsto \quad E_{\mathrm{P}}(\omega)=\sum_{m=1}^{M} \omega^{1-m} c_{0}^{[2 m]} .
$$

In Table IV, we show the partial sums up to the order $M$ with $m_{c}-10<M \leq m_{c}$. The critical order $m_{c}$ for all the cases $\omega=30,50,70,90$, and 110 are given by $m_{c}=\omega / 2$.

TABLE IV: The first finite partial sums of the perturbative corrections.

\begin{tabular}{|c|c|c|c|c|c|c|c|}
\hline \multicolumn{2}{|r|}{$\omega=30$} & \multicolumn{4}{|c|}{$\omega=50$} & \multicolumn{2}{|r|}{$\omega=70$} \\
\hline$M$ & $\sum_{m=1}^{M} \omega^{1-m} c_{0}^{[2 m]}$ & $M$ & \multicolumn{3}{|c|}{$\sum_{m=1}^{M} \omega^{1-m} c_{0}^{[2 m]}$} & M & $\sum_{m=1}^{M} \omega^{1-m} c_{0}^{[2 m]}$ \\
\hline 6 & $-0.821307 \cdots$ & 16 & \multicolumn{3}{|c|}{$-0.7883969801 \cdots$} & 26 & -0.776334145649363 \\
\hline 7 & -0.821522 & 17 & \multicolumn{3}{|c|}{$-0.7883969881 \cdots$} & 27 & -0.7763 \\
\hline 8 & -0.8 & 18 & \multicolumn{3}{|c|}{$-0.7883969939 \ldots$} & 28 & 0061 . \\
\hline 9 & $-0.821714 \cdots$ & 19 & \multicolumn{3}{|c|}{$-0.7883969983 \ldots$} & 29 & $50315 \ldots$ \\
\hline 10 & $-0.821764 \cdots$ & 20 & \multicolumn{3}{|c|}{$-0.7883970018 \cdots$} & 30 & $50532 \ldots$ \\
\hline 11 & $-0.821800 \cdots$ & 21 & \multicolumn{3}{|c|}{$-0.7883970047 \cdots$} & 31 & $4145650723 \cdots$ \\
\hline 12 & -0.8 & 22 & \multicolumn{3}{|c|}{$-0.7883970072 \ldots$} & 32 & -0.776 \\
\hline 13 & -0.821856 . & 23 & \multicolumn{3}{|c|}{$-0.7883970095 \ldots$} & 33 & -0.776334145651060 \\
\hline 14 & $-0.821880 \cdots$ & 24 & \multicolumn{3}{|c|}{$-0.7883970118 \ldots$} & 34 & $-0.776334145651217 \cdots$ \\
\hline$m_{c}$ & -0.821903 & $m_{c}$ & \multicolumn{3}{|c|}{$-0.7883970140 \cdots$} & $m_{c}$ & -0.7763 \\
\hline \multicolumn{4}{|c|}{$\omega=90$} & \multicolumn{4}{|r|}{$\omega=110$} \\
\hline M & \multicolumn{3}{|c|}{$\sum_{m=1}^{M} \omega^{1-m} c_{0}^{[2 m]}$} & \multicolumn{4}{|c|}{$\sum_{m=1}^{M} \omega^{1-m} c_{0}^{[2 m]}$} \\
\hline 36 & \multicolumn{3}{|c|}{$-0.7700548367611132120 \cdots$} & 46 & \multicolumn{3}{|c|}{$-0.7661975631223371561012 \cdots$} \\
\hline 37 & \multicolumn{3}{|c|}{$-0.7700548367611132318 \ldots$} & 47 & \multicolumn{3}{|c|}{$-0.7661975631223371561022 \ldots$} \\
\hline 38 & \multicolumn{3}{|c|}{$-0.7700548367611132485 \ldots$} & 48 & \multicolumn{3}{|c|}{$-0.7661975631223371561031 \ldots$} \\
\hline 39 & \multicolumn{3}{|c|}{$-0.7700548367611132628 \ldots$} & 49 & \multicolumn{3}{|c|}{$-0.7661975631223371561039 \ldots$} \\
\hline 40 & \multicolumn{3}{|c|}{$-0.7700548367611132755 \cdots$} & 50 & \multicolumn{3}{|c|}{$-0.7661975631223371561046 \cdots$} \\
\hline 41 & \multicolumn{3}{|c|}{$-0.7700548367611132871 \cdots$} & $51-$ & \multicolumn{3}{|c|}{$-0.7661975631223371561053 \cdots$} \\
\hline 42 & \multicolumn{3}{|c|}{$-0.7700548367611132978 \ldots$} & $52-$ & \multicolumn{3}{|c|}{$-0.7661975631223371561059 \ldots$} \\
\hline 43 & \multicolumn{3}{|c|}{$-0.7700548367611133080 \ldots$} & $53-$ & -0. & 66197 & $75631223371561065 \cdots$ \\
\hline 44 & $4-0.770054836$ & & $33179 \cdots$ & 54 & & & $75631223371561071 \cdots$ \\
\hline$m_{c}$ & $c \quad-0.770054836$ & & $33277 \cdots$ & $m_{c}$ & -0. & 6197 & $75631223371561077 \cdots$ \\
\hline
\end{tabular}

Comparing the results in Table IV with the ones in Table III we easily see that the asymptotic values of the perturbative corrections for all the cases are in good agreement 
with the values $E_{\mathrm{P}}^{\mathrm{ST}}(\omega)$ predicted by our formula (8a) but apparently deviate from the values $E_{\mathrm{P}}^{\mathrm{AL}}(\omega)$ predicted by the Alhendi and Lashin's formula (3a).

We note that the differences between $E_{\mathrm{P}}^{\mathrm{ST}}(\omega)$ and $\sum_{m=1}^{M} \omega^{1-m} c_{0}^{[2 m]}$ are minimum around $M \sim m_{c}-1$. It indicates that $\operatorname{Re} E_{\mathrm{P}}(g)$ in the case has the perturbation series as strong asymptotic series [10], that is, there exist positive real constants $C$ and $\sigma$ so that

$$
\left|\operatorname{Re} E_{\mathrm{P}}(g)-\sum_{m=1}^{M} g^{2 m} c_{0}^{[2 m]}\right| \leq C \sigma^{M+1}(M+1) !\left|g^{2}\right|^{M+1},
$$

for all $M$ and all $g^{2}>0$ near the origin. Indeed, if it is the case, we have with the aid of Eq. (17a) and $\sigma=2$,

$$
\left|\operatorname{Re} E_{\mathrm{P}}(g)-\sum_{m=1}^{M} g^{2 m} c_{0}^{[2 m]}\right| \lesssim C^{\prime}(M+7 / 4)^{1 / 4}\left|g^{2(M+1)} c_{0}^{[2(M+1)]}\right|,
$$

where $C^{\prime}=C \pi \Gamma(3 / 4) / 2^{5 / 4}$. The right hand side is minimum around $M \sim m_{c}-1$ by the definition of the critical order $m_{c}$, and thus the above fact would be naturally understood.

Finally, we would like to mention about the fact that in the parameter region we have examined, $30 \leq \omega \leq 110$ or $0.095 \lesssim g \lesssim 0.18$, the following relation is roughly satisfied:

$$
\min _{m}\left|g^{2 m} c^{[2 m]}\right|=\left|g^{2 m_{c}} c^{\left[2 m_{c}\right]}\right| \sim\left|\operatorname{Re} E_{\mathrm{NP}}(g)\right| \times 10^{-1}
$$

Interestingly, we can show that a similar relation is observed generically as far as the system under consideration has a nonperturbative effect. Suppose the following conditions are satisfied for smaller values of the coupling constant $g^{2}$ involved in the system under consideration:

$$
\operatorname{Im} E_{\mathrm{NP}}(g) \sim C g^{-2(\nu+1)} \mathrm{e}^{-1 / b g^{2}}, \quad \operatorname{Re} E_{\mathrm{NP}}(g)=A \operatorname{Im} E_{\mathrm{NP}}(g),
$$

where $A, C$, and $b>0$ are real constants. Then, we can prove the following intriguing relation for smaller $g^{2}$ :

$$
\min _{m}\left|g^{2 m} c^{[2 m]}\right| \sim \sqrt{\frac{2 b \mathrm{e}}{A^{2} \pi}}\left|g \operatorname{Re} E_{\mathrm{NP}}(g)\right| .
$$

For the proof, we first note that the first condition in Eq. (24) implies

$$
c^{[2 m]} \sim-\frac{C}{\pi} b^{m+\nu+1} \Gamma(m+\nu+1) \equiv \bar{c}^{[2 m]},
$$

for larger $m$. Next, we define a function $f$ by

$$
f(\mu ; g) \equiv\left|g^{2 \mu} \bar{c}^{[2 \mu]}\right|
$$

It is evident that for larger integer $m$ the function $f(m ; g)$ well approximates the magnitude of the $m$ th-order perturbative correction. The derivative with respect to $\mu$ reads,

$$
\frac{\partial}{\partial \mu} f(\mu ; g)=f(\mu ; g)\left[\ln \left(b g^{2}\right)+\psi(\mu+\nu+1)\right]
$$


where $\psi$ denotes the digamma function. Hence $f(\mu ; g)$ takes minimum value at $\mu=\bar{\mu}, \bar{\mu}$ satisfying

$$
\ln \left(b g^{2}\right)+\psi(\bar{\mu}+\nu+1)=0 .
$$

For smaller value of $g^{2} \ll 1$, we see $\bar{\mu}$ becomes larger. Thus, applying the asymptotic expansion of the digamma function [11]:

$$
\psi(z) \sim \ln z-\frac{1}{2 z}+O\left(z^{-2}\right),
$$

we obtain

$$
b g^{2} \sim \frac{1}{\bar{\mu}+\nu+1} \exp \left(\frac{1}{2(\bar{\mu}+\nu+1)}\left[1+O\left(\bar{\mu}^{-1}\right)\right]\right) .
$$

With the aid of the Stirling formula and Eq. (31), we have

$$
\Gamma(\bar{\mu}+\nu+1) \sim \sqrt{2 \pi} \mathrm{e}^{1 / 2}\left(b g^{2}\right)^{-(\bar{\mu}+\nu+1 / 2)} \mathrm{e}^{-1 / b g^{2}}\left[1+O\left(\bar{\mu}^{-1}\right)\right] .
$$

Therefore, the minimum value of $f(\mu, g)$, which would provide a good approximation to the minimum magnitude of the perturbative correction at the critical order $m_{c} \sim \bar{\mu}$, is estimated as,

$$
\begin{aligned}
f(\bar{\mu} ; g) & =\frac{b^{\nu+1}}{\pi}|C|\left(b g^{2}\right)^{\bar{\mu}} \Gamma(\bar{\mu}+\nu+1) \\
& \sim \sqrt{\frac{2 b \mathrm{e}}{\pi}}|C| g^{-2(\nu+1 / 2)} \mathrm{e}^{-1 / b g^{2}}\left[1+O\left(\bar{\mu}^{-1}\right)\right] \\
& \sim \sqrt{\frac{2 b \mathrm{e}}{\pi}}\left|A^{-1} g \operatorname{Re} E_{\mathrm{NP}}(g)\right|\left[1+O\left(\bar{\mu}^{-1}\right)\right],
\end{aligned}
$$

and thus we obtain the relation (25). In our case $A=1$ and $b=2$, and thus the relation (23) for $g \sim 0.1$ follows. As a consequence, we also find that the next-order nonperturbative corrections of order $O\left(g^{2} \operatorname{Re} E_{\mathrm{NP}}\right)$ becomes negligible in comparison with the perturbative correction for sufficiently small $g$; from the relation (25) we readily obtain

$$
O\left(g^{2} \operatorname{Re} E_{\mathrm{NP}}\right) \sim O\left(g \min _{m}\left|g^{2 m} c^{[2 m]}\right|\right) .
$$

Therefore, the next-order nonperturbative corrections do not affect the analysis for $g \sim 0.1$ in this section.

\section{WKB CALCULATION}

So far, we have checked the accuracy of the semi-classical calculations of the path-integral by comparing with the exact values calculated numerically. In this section, we make a comparison in a different way. To this end, we employ another nonperturbative approach to derive formulas for the same physical quantities. The method we shall use here is the WKB approximation for the Schrödinger equation. In the following, we shall always consider 
the leading terms of the power expansion in $g$ since we are interested in the quantization condition for the nonperturbative contribution.

Let us consider the more general Hamiltonian (44) for all $\epsilon g^{2} \ll 1$. The system has parity symmetry and thus it is sufficient to study the connection condition of the WKB wave function only on the half-line $q \in(0, \infty)$. In the vicinity of the minimum of the central potential well, the Schrödinger equation for the Hamiltonian (4) in the leading order of $g$ is given by

$$
\left(-\frac{1}{2} \frac{\mathrm{d}^{2}}{\mathrm{~d} q^{2}}+\frac{1}{2} q^{2}\right) \psi(q)=\left(E-\frac{\epsilon}{2}\right) \psi(q)
$$

The local solutions possessing a definite parity \pm are expressed as

$$
\psi(q)=A_{ \pm}\left(D_{\nu}(-\sqrt{2} q) \pm D_{\nu}(\sqrt{2} q)\right),
$$

where $A_{ \pm}$are constants and $D_{\nu}$ is the parabolic cylinder function with $\nu=E-\epsilon / 2-1 / 2$. In a similar way, around the minimum of the right side potential well, the Schrödinger equation is approximated by

$$
\left[-\frac{1}{2} \frac{\mathrm{d}^{2}}{\mathrm{~d} q^{2}}+2\left(q-\frac{1}{g}\right)^{2}\right] \psi(q)=(E+\epsilon) \psi(q) .
$$

and the local solution which vanishes at $q \rightarrow \infty$ is given by

$$
\psi(q)=B D_{\lambda}(2(q-1 / g)),
$$

where $B$ is a constant and $\lambda=E / 2+\epsilon / 2-1 / 2$. The solutions (36) and (38) are to be connected with the following WKB solution in the classically forbidden region $\left(q_{1} \ll q \ll q_{2}\right)$ :

$$
\psi(q)=\frac{C_{1}}{k(q)^{1 / 2}} \exp \left(-\int_{q_{1}}^{q} \mathrm{~d} x k(x)\right)+\frac{C_{2}}{k(q)^{1 / 2}} \exp \left(\int_{q_{1}}^{q} \mathrm{~d} x k(x)\right),
$$

where

$$
k(x)=\sqrt{x^{2}\left(1-g^{2} x^{2}\right)^{2}+\epsilon\left(1-3 g^{2} x^{2}\right)-2 E} .
$$

The positive classical turning points $q_{i}(i=1,2)$ with $0<q_{1}<q_{2}$ defined by the solutions of $V\left(q_{i}\right)=E$ are,

$$
q_{1}=\sqrt{2 E-\epsilon}+O\left(g^{2}\right), \quad q_{2}=\frac{1}{g}-\sqrt{\frac{E+\epsilon}{2}}+O(g) .
$$

In order to connect the wave functions obtained in the each region, it is important to note that the leading term in $g$ of the WKB solution (39) varies according to the position it is viewed from. If it is viewed from the point around the central potential well, the integral in the exponent in Eq. (39) is evaluated as

$$
\begin{aligned}
\int_{q_{1}}^{q} \mathrm{~d} x k(x) & =\frac{1}{g^{2}} \int_{g q_{1}}^{g q} \mathrm{~d} \omega \sqrt{w^{2}\left(1-w^{2}\right)^{2}+\epsilon g^{2}\left(1-3 w^{2}\right)-2 E} \\
& =\frac{1}{g^{2}} \int_{g q_{1}}^{g q} \mathrm{~d} \omega\left[w\left(1-w^{2}\right)-\frac{1}{2} \frac{(2 E-\epsilon) g^{2}}{w\left(1-w^{2}\right)}-\frac{3 \epsilon g^{2}}{2} \frac{w}{1-w^{2}}+\cdots\right] \\
& =\left[\frac{\omega^{2}}{2 g^{2}}-\frac{\omega^{4}}{4 g^{2}}+\frac{\epsilon-2 E}{2} \ln |\omega|+\frac{\epsilon+E}{2} \ln \left|1-\omega^{2}\right|+\cdots\right]_{g q_{1}}^{g q},
\end{aligned}
$$


and $k(q) \sim q+\cdots$. Thus, in the leading order of $g$ we obtain the WKB wave function as

$$
\psi(q) \sim \frac{C_{1}}{q^{1 / 2}} \mathrm{e}^{-q^{2} / 2}\left(\frac{\mathrm{e} q}{\sqrt{2 E-\epsilon}}\right)^{(2 E-\epsilon) / 2}+\frac{C_{2}}{q^{1 / 2}} \mathrm{e}^{q^{2} / 2}\left(\frac{\mathrm{e} q}{\sqrt{2 E-\epsilon}}\right)^{-(2 E-\epsilon) / 2} .
$$

Comparing this with the following asymptotic form for $q \gg 1$ of the wave function (36) (cf. Ref. [11]) determined in the region of the central potential well:

$$
\psi(q) \sim A_{ \pm}\left[\left((-1)^{\nu} \pm 1\right) \mathrm{e}^{-q^{2} / 2}(\sqrt{2} q)^{\nu}+\frac{\sqrt{2 \pi}}{\Gamma(-\nu)} \mathrm{e}^{q^{2} / 2}(\sqrt{2} q)^{-\nu-1}\right]
$$

we have the following connection condition:

$$
\frac{\sqrt{2 \pi}}{\left((-1)^{\nu} \pm 1\right) \Gamma(-\nu)}=\frac{C_{2}}{C_{1}}\left(\frac{2 \sqrt{E-\epsilon / 2}}{\mathrm{e}}\right)^{2 E-\epsilon} .
$$

On the other hand, the integral in the exponent in Eq. (39) viewed from the point around the right side potential well is evaluated as

$$
\begin{aligned}
& \int_{q_{1}}^{q} \mathrm{~d} x k(x)=\frac{1}{g^{2}} \int_{g q_{1}-1}^{g q-1} \mathrm{~d} \omega \sqrt{\omega^{2}(1+\omega)^{2}(2+\omega)^{2}-\epsilon g^{2}\left(2+6 \omega+3 \omega^{2}\right)-2 E g^{2}} \\
& =-\frac{1}{g^{2}} \int_{g q_{1}-1}^{g q-1} \mathrm{~d} \omega\left[\omega(1+\omega)(2+\omega)-\frac{\epsilon g^{2}\left(2+6 \omega+3 \omega^{2}\right)+2 E g^{2}}{2 \omega(1+\omega)(2+\omega)}+\cdots\right] \\
& =\left[-\frac{1}{g^{2}}\left(\omega^{2}+\omega^{3}+\frac{\omega^{4}}{4}\right)+\frac{\epsilon+E}{2} \ln |\omega(2+\omega)|+\frac{\epsilon-2 E}{2} \ln |1+\omega|+\cdots\right]_{g q_{1}-1}^{g q-1},
\end{aligned}
$$

and $k(q) \sim 1 / g-q+\cdots$. Thus, in the leading order of $g$ we obtain the WKB wave function as

$$
\begin{aligned}
\psi(q) \sim & \frac{C_{1} \mathrm{e}^{-1 / 4 g^{2}} g^{-3 E / 2}}{(1 / g-q)^{1 / 2}} \mathrm{e}^{(q-1 / g)^{2}}(2(1 / g-q))^{-(E+\epsilon) / 2}\left(\frac{\mathrm{e}}{\sqrt{2 E-\epsilon}}\right)^{(2 E-\epsilon) / 2} \\
& +\frac{C_{2} \mathrm{e}^{1 / 4 g^{2}} g^{3 E / 2}}{(1 / g-q)^{1 / 2}} \mathrm{e}^{-(q-1 / g)^{2}}(2(1 / g-q))^{(E+\epsilon) / 2}\left(\frac{\mathrm{e}}{\sqrt{2 E-\epsilon}}\right)^{-(2 E-\epsilon) / 2} .
\end{aligned}
$$

Matching this with the following asymptotic form for $1 / g-q \gg 1$ of the wave function (38) determined in the region of the right side potential well:

$$
\psi(q) \sim B\left[\mathrm{e}^{-(q-1 / g)^{2}}(2(q-1 / g))^{\lambda}-\frac{\sqrt{2 \pi}(-1)^{\lambda}}{\Gamma(-\lambda)} \mathrm{e}^{(q-1 / g)^{2}}(2(q-1 / g))^{-\lambda-1}\right]
$$

we get another connection condition as follows:

$$
-\frac{\sqrt{2 \pi}}{(-1)^{\lambda+1} \Gamma(-\lambda)}=\frac{C_{1}}{C_{2}} \mathrm{e}^{-1 / 2 g^{2}} g^{-3 E}\left(\frac{\mathrm{e}}{\sqrt{2 E-\epsilon}}\right)^{2 E-\epsilon} .
$$


Therefore, eliminating the coefficient $C_{1} / C_{2}$ in Eqs. (45) and (49), we finally obtain the following quantization condition:

$$
\begin{aligned}
\frac{\sqrt{2}}{\pi g^{2}} \mathrm{e}^{-1 / 2 g^{2}} \frac{(-1)^{E-1 / 2-\epsilon / 2} \pm 1}{2} & \left(\frac{2}{g^{2}}\right)^{E-1 / 2-\epsilon / 2} \Gamma\left(-E+\frac{1}{2}+\frac{\epsilon}{2}\right) \\
& \times\left(-\frac{1}{g^{2}}\right)^{E / 2-1 / 2+\epsilon / 2} \Gamma\left(-\frac{E}{2}+\frac{1}{2}-\frac{\epsilon}{2}\right)=1 .
\end{aligned}
$$

Strikingly, this condition is in complete agreement with the one obtained previously by us with the valley method, Eq. (5.19) in Ref. 4], from which our formulas of the nonperturbative effect (5) - (6) have been derived. We note that the coincidence is achieved not only for the case $\epsilon=0$ we have examined in the preceding sections but also for all $\epsilon \neq 0$ where we cannot apply the usual instanton technique since the classical configuration now becomes a bounce solution.

\section{SUMMARY}

In this letter, we have extensively examined lowest three energies of the symmetric triplewell potential with non-equivalent vacua by comparing the two different sets of the formulas, the one is calculated by means of an instanton technique with the dilute gas approximation in Ref. 7] and the other is by the valley method beyond this approximation scheme in Ref. [4].

First, we compared numerically both the formulas with the exact values for the spectral splitting between the first and second excited states due to the purely nonperturbative effect. We have found that in contrast to the latter formulas the prediction of the former formulas get worse as the value of the parameter tends to the region where the semi-classical methods should work better. Thus, contrary to folklore that this kind of problem can be handled by the use of the dilute gas approximation, our careful comparison with the exact results indicates that the dilute gas approximation is insufficient to produce the correct asymptotic formulas even if we restrict ourselves to examining the low lying eigenvalues.

Furthermore, we examined for the ground state both the perturbative and nonperturbative contributions. We have found that the asymptotic values of the perturbative corrections calculated from the perturbation series deviate from the predicted values obtained from the instanton calculation with the dilute gas approximation but are in good agreement with the ones obtained from the valley method calculation.

We also checked the accuracy of the imaginary parts of the latter formulas by testing the prediction on the large order behavior of the perturbation series. We evaluated the perturbative coefficients up to the order 300 and compared them with the predicted asymptotic behaviors. We have confirmed the correctness of the prediction for both the ground and excited states.

Finally, we carried out the WKB calculation in the leading order of the coupling constant. The resulting WKB quantization condition is in complete agreement with the one obtained by means of the valley method in Ref. [4], from which our formulas have been derived. In other words, the dilute gas approximation in the path integral formalism does not correspond to a proper semi-classical approximation. In this respect, we would like to recall the fact that discrepancy between the dilute gas approximation and the WKB approximation has 
been already reported in Ref. [12] for the wave functions even in the case of the symmetric double-well potential.

To conclude, all the present analyses entirely support the valley method calculation and indicate the limitation of the dilute gas approximation in the present triple-well potential

problem. Therefore, we would like to repeat the assertion in Ref. [4] that the applicability of the dilute gas approximation would be quite limited.

\section{Acknowledgments}

We would like to thank the organizers of the international conference "New Frontiers in Quantum Mechanics" (July 5-8, 2004, Shizuoka University, Japan) where the present work started. This work was partially supported by the Grand-in-Aid for Scientific Research No.14740158 (M. S.) and by a Spanish Ministry of Education, Culture and Sports research fellowship (T. T.).

[1] S. Colemann, Aspects of Symmetry (Cambridge Univ. Press, 1985).

[2] S.-Y. Lee, J.-R. Kahng, S.-K. Yoo, D. K. Park, C. H. Lee, C. S. Park, and E.-S. Yim, Mod. Phys. Lett. A12, 1803 (1997), quant-ph/9608015.

[3] J. Casahorrán, Phys. Lett. A283, 285 (2001), quant-ph/0103010.

[4] M. Sato and T. Tanaka, J. Math. Phys. 43, 3484 (2002), hep-th/0109179.

[5] H. Aoyama, H. Kikuchi, I. Okouchi, M. Sato, and S. Wada, Nucl. Phys. B553, 644 (1999), hep-th/9808034.

[6] H. Aoyama, M. Sato, and T. Tanaka, Nucl. Phys. B619, 105 (2001), quant-ph/0106037.

[7] H. A. Alhendi and E. I. Lashin, Mod. Phys. Lett. A19, 2103 (2004), quant-ph/0406200.

[8] J. C. L. Guillou and J. Zinn-Justin, eds., Large-Order Behavior of Perturbation Theory (North Holland, 1990).

[9] C. M. Bender and T. T. Wu, Phys. Rev. 184, 1231 (1969).

[10] M. Reed and B. Simon, Methods of Modern Mathematical Physics IV: Analysis of Operators (Academic Press, New York, 1978).

[11] I. S. Gradshteyn and I. M. Ryzhik, Table of Integrals, Series, and Products (Academic Press, San Diego, 2000), sixth ed.

[12] G. C. Rossi and M. Testa, Ann. Phys. 148, 144 (1983). 